\title{
On Estimating the Robust Domain of Attraction for Uncertain Non-Polynomial Systems: An LMI Approach
}

\author{
Dongkun Han, and Matthias Althoff
}

\begin{abstract}
An increasingly important issue in the area of uncertain systems is the estimation of the Robust Domain of Attraction (RDA). Though this topic is of great interest, most of attention has been paid to the RDA for uncertain polynomial systems. This paper considers the RDA for rational polynomial systems and non-polynomial systems, both with parametric uncertainties, which are constrained in a semialgebraic set. The main underlying idea is to reformulate the original system to an uncertain rational polynomial system by using the truncated Taylor expansion and the parameterizable remainder of nonpolynomial functions. A novel way to compute the largest estimate of the RDA is proposed by using a given rational Lyapunov function and the squared matrix representation technique (SMR). Lastly, the benefits of this approach are presented by a numerical example.
\end{abstract}

\section{INTRODUCTION}

It is well known that estimating the RDA of an equilibrium point is an interesting yet challenging problem for uncertain systems. In fact, the RDA is usually a complicated set, which is difficult to be expressed by an analytic representation even for the uncertainty-free case [1]. In order to compute the exact domain of attraction, some elegant methods are proposed, e.g., the Zubov equation method and the maximal Lyapunov function method [2]. However, the solution of the Zubov equation and the maximal Lyapunov function are not easy to be found in most cases. Fortunately, an under-estimation method has been proven to be effective by using the sublevel set of Lyapunov functions [1]. Especially over the last decade, thanks to the rapid developments of the Sum of Squares (SOS) technique and the semialgebraic geometry [3]-[5], increasingly efficient methods have been proposed based on semidefinite programming and Linear Matrix Inequalities (LMI) [6]-[10].

An overwhelming majority of existing works is concerned with polynomial systems, while recently there are two new trends. The first trend is to study the RDA of uncertain systems with parametric uncertainty, which is stimulated by practical needs, e.g., circuit design with changing parameters influenced by temperature, and stabilization of mechanical system with time-varying loads [11]-[13]. In [11], the RDA is computed for polynomial systems with bounded parametric uncertainties by using polynomial Lyapunov functions and a branch-and-bound type procedure. By also using a Lyapunov function method, the largest estimate of the RDA for polynomial systems is computed by an SOS decomposition [14], [15]. On the other hand, the demands for developments

The authors are with the Institut für Informatik, Technische Universität München, Boltzmannstraße 3, 85748 Garching bei, München, Germany. E-mail: dongkun.han@tum.de. targeting non-polynomial systems and rational systems are increasingly high, considering the practical requests in numerous areas, e.g., for non-polynomial systems in robotic arm modeling [16], airplanes in longitude flight [17], for rational systems in enzyme-catalyzed interaction [18], and metabolic networking organization [19]. With regard to nonpolynomial systems, researchers are interested in polynomial approximation methods, like replacing the nonlinear terms with new variables and recasting the state space to an expanded one [17], covering the non-polynomial functions into a convex hull of a group of polynomials [20]. In [21], an approach is provided by using Chebychev points with a chosen quadratic Lyapunov function for the uncertainty-free case. Related to this work is the method of [22], where a rational Lyapunov function is used to estimate the RDA of uncertain polynomial systems. Unfortunately, the problem, estimating the RDA for non-polynomial systems and for rational polynomial systems with parametric uncertainties, has never been considered, to the best knowledge of the authors.

Motivated from the above, this work extends the result of [22], [23] to the case of uncertain rational polynomial and non-polynomial systems, and contributes in the following three aspects:

- For the first time, the RDA is computed for both, an uncertain model of rational polynomial functions and a class of nonlinear functions with truncated Taylor expansion, under the effect of parametric uncertainty, which is constrained in a semialgebraic set.

- By introducing a new class of parameter-dependent SOS and employing the SMR technique, a quasi-convex optimization problem is formulated to compute the largest estimate of the RDA via expanding the sublevel set of a rational Lyapunov function.

- A necessary and sufficient condition is provided for the tightness of the computed lower bound of the RDA. This tightness can be established by a method which first verifies whether a power vector is in a null space and then verifies the vanishing of the derivative of the selected Lyapunov function.

\section{PRELIMINARIES}

Notations: $\mathbb{N}^{n}, \mathbb{R}^{n}$ : natural and real number sets with dimension $n ; \mathbb{R}^{+}$: positive real number set; $0_{n}$ : origin of $\mathbb{R}^{n}$; $\mathbb{R}_{0}^{n}: \mathbb{R}^{n} \backslash\left\{0_{n}\right\} ; A_{i, j}$ : element in the $i$-th row and in the $j$-th column of matrix $A ; A^{T}$ : transpose of $A ; A>0(A \geq 0)$ : symmetric positive definite (semidefinite) matrix $A ; A \otimes B$ : 
Kronecker product of matrices $A$ and $B ; A \circ B$ : elementwise product (Hadamard Product) of matrices $A$ and $B$ of the same dimension, i.e., $(A \circ B)_{i, j}=A_{i, j} \cdot B_{i, j} ; A \oslash B$ : elementwise division of matrices $A$ and $B$ of the same dimension, i.e., $(A \oslash B)_{i, j}=A_{i, j} / B_{i, j} ; \operatorname{ker}(A)$ : null space of linear map $A$; $\operatorname{deg}_{x}(f)$ : degree of polynomial function $f(x)$ in $x ; \nabla f$ : gradient of $f(x)$, i.e., $\nabla f=\left(\frac{\partial f}{\partial x_{1}}, \ldots, \frac{\partial f}{\partial x_{n}}\right)^{T}$; $\operatorname{lcm}(v)$ : the least common multiple of all the entries of vector $v \in \mathbb{R}^{n}$; $\operatorname{diag}(v)$ : a square diagonal matrix with the elements of vector $v \in \mathbb{R}^{n}$ on the main diagonal; $(*)^{T} A B$ in a form of SMR: $B^{T} A B$.

Let $\mathcal{P}$ be the set of polynomials and $\mathcal{P}^{n \times m}$ be the set of matrix polynomials with dimension $n \times m$. A polynomial $p(x) \in \mathcal{P}$ is nonnegative if $p(x) \geq 0$ for all $x \in \mathbb{R}^{n}$. An effective way of checking whether $p(x)$ is nonnegative consists of checking whether $p(x)$ can be expressed as an SOS, i.e., $p(x)=\sum_{i=1}^{l} p_{i}(x)^{2}$ for some $p_{1}, \ldots, p_{l} \in \mathcal{P}$. We denote the set of SOS polynomials as $\mathcal{P}^{\mathrm{SOS}}$. If $p(x) \in \mathcal{P}^{\mathrm{SOS}}$ becomes 0 only for $x=0_{n}$ and $p(x)$ is without monomials of degree 0 and 1 , we call $p(x)$ local SOS which is denoted by $\mathcal{P}_{0}^{\mathrm{SOS}}$.

\section{A. Model Formulation}

In this paper, we consider the following model:

$$
\dot{x}(t)=f(x(t), \theta)+\sum_{i=1}^{r} g_{i}(x(t), \theta) \zeta_{i}\left(x_{a_{i}}(t)\right), x \in \mathcal{D}
$$

where $\mathcal{D} \subseteq \mathbb{R}^{n}$ is the domain, $x \in \mathbb{R}^{n}$ denotes the state vector, $x(0)=x_{\text {init }} \in \mathbb{R}^{n}$ is the initial state, $\theta \in \mathbb{R}^{n_{\theta}}$ denotes the uncertain parameter vector, $f(x(t), \theta) \in \mathbb{R}^{n}$, are vector rational polynomial functions which can be expressed as

$$
\begin{aligned}
f(x(t), \theta) & =f_{\text {num }}(x(t), \theta) \oslash f_{\operatorname{den}}(x(t), \theta), \\
g_{i}(x(t), \theta) & =g_{\text {num }_{i}}(x(t), \theta) \oslash g_{\operatorname{den}_{i}}(x(t), \theta),
\end{aligned}
$$

in which $\oslash$ denotes the element-wise division, $f_{\text {num }}(x(t), \theta)$, $f_{\mathrm{den}}(x(t), \theta), g_{\mathrm{num}_{i}}(x(t), \theta), g_{\mathrm{den}_{i}}(x(t), \theta) \in \mathcal{P}^{n}$ are vector polynomial functions, $\zeta_{1}\left(x_{a_{i}}(t)\right), \ldots, \zeta_{r}\left(x_{a_{i}}(t)\right)$ denote nonpolynomial functions and $a_{1}, \ldots, a_{r} \in\{1, \ldots, n\}$ are the indexes, satisfying $r<n$. The uncertain parameter $\theta$ is in a semialgebraic set

$$
\Theta=\left\{\theta \in \mathbb{R}^{n_{\theta}}: a(\theta) \geq 0, b(\theta)=0\right\}
$$

where $a(\theta): \mathbb{R}^{n_{\theta}} \rightarrow \mathbb{R}^{n_{a}}$ and $b(\theta): \mathbb{R}^{n_{\theta}} \rightarrow \mathbb{R}^{n_{b}}$ are polynomial functions. For the brevity of the presentation, the dependence of functions on time $t$, state $x(t)$ and parameter $\theta$ will be omitted whenever reasonable.

In this paper, we are interested in estimating the RDA of an equilibrium point. First, we introduce the definition of the RDA of the origin [11], [13], i.e.,

$$
\mathcal{R}=\bigcap_{\theta \in \Theta}\left\{x_{\text {init }} \in \mathbb{R}^{n}: \lim _{t \rightarrow+\infty} \chi\left(t ; x_{\text {init }}, \theta\right)=0_{n}\right\},
$$

where $\chi\left(t ; x_{\mathrm{init}}, \theta\right)$ denotes the solution of system (1) at time $t$, starting from initial state $x_{i n i t}$ and using parameter $\theta$.

In addition, we assume that $\zeta_{i}, i=1, \ldots, r$, are $k$ times differentiable at the origin and $k+1$ times differentiable within the open interval $\left(0, x_{a_{i}}\right)$, then $\zeta_{i}$ could be rewritten by a Taylor expansion evaluated at the origin as follows:

$$
\zeta_{i}\left(x_{a_{i}}\right)=\eta_{i}\left(x_{a_{i}}\right)+\xi_{i} \frac{x_{a_{i}}^{k+1}}{(k+1) !}
$$

where $\xi_{i} \in \mathbb{R}$ is a bounded parameter, $k$ denotes the truncation degree and $\eta_{i}\left(x_{a_{i}}\right)$ is the $k$-th order Taylor polynomial:

$$
\eta_{i}\left(x_{a_{i}}\right)=\left.\sum_{j=0}^{k} \frac{d^{j} \zeta_{i}\left(x_{a_{i}}\right)}{d x_{a_{i}}^{j}}\right|_{x_{a_{i}}=0} \frac{x_{a_{i}}^{j}}{j !} .
$$

Remark 1: We exploit the parameters $\xi_{i}$ to overapproximate the Taylor remainder $\zeta_{i}\left(x_{a_{i}}\right)-\eta_{i}\left(x_{a_{i}}\right)$, where $\xi=\left(\xi_{1}, \ldots, \xi_{r}\right)^{T}$ is in the orthotope

$$
\Xi=\left[\underline{\tau}_{1}, \bar{\tau}_{1}\right] \times \cdots \times\left[\underline{\tau}_{r}, \bar{\tau}_{r}\right]
$$

and $\underline{\tau}_{i}, \bar{\tau}_{i} \in \mathbb{R}, i=1, \ldots, r$, are selected as the tightest bounds fulfilling

$$
\underline{\tau}_{i} \leq\left.\frac{d^{k+1} \zeta_{i}\left(x_{a_{i}}\right)}{d x_{a_{i}}^{k+1}}\right|_{x_{a_{i}=\iota}} \leq \bar{\tau}_{i}
$$

for all $\iota \in \mathcal{I}$, where $\mathcal{I}$ is a set chosen in the sublevel set of a Lyapunov function $\mathcal{V}_{x_{a_{i}}}$ which will be introduced in the next subsection.

Remark 2: The model (1) is a quite general one, including a sum of products between a group of parameter-dependent rational functions and a group of non-polynomial functions $\zeta_{i}$ which are expressed by Taylor expansion with a parameterized remainder in Lagrange form. Previous models considered for computing the domain of attraction [8], [11], [13], [16], [17] are thus special cases of the model considered in this work.

Example 1: Consider a deliberately simple 2-dimensional system described by

$$
\left\{\begin{array}{l}
\dot{x}_{1}=\frac{-x_{1}-5 x_{2}^{5}-x_{1}^{2}\left(\theta_{1}+\theta_{2}\right)}{1+x_{2}^{2}}-x_{2} \sin \left(x_{1}\right) \\
\dot{x}_{2}=\frac{1}{\theta_{1}}-2 x_{2} \theta_{2}-2 x_{1}^{3}-\frac{e^{x_{2}}}{\theta_{1}}
\end{array}\right.
$$

with $1 \leq \theta_{1} \leq 2,0 \leq \theta_{2} \leq 1$ and $\theta_{1}^{2}+\theta_{2}^{2}-2=0$. We can write this model in the form of (1) by choosing $f_{\text {num }}=\left(-x_{1}-5 x_{2}^{5}-x_{1}^{2}\left(\theta_{1}+\theta_{2}\right),-2 x_{2} \theta_{2}-2 x_{1}^{3}\right)^{T}, f_{\text {den }}=$ $\left(1+x_{2}^{2}, 1\right)^{T}, g_{1}=\left(x_{2}, 0\right)^{T}, g_{2}=\left(0, \frac{1}{\theta_{1}}\right)^{T}, \zeta_{1}=\sin \left(x_{1}\right)$, $\zeta_{2}=1-e^{x_{2}}, a(\theta)=\left(\theta_{1}-1,2-\theta_{1}, \theta_{2}, 1-\theta_{2}\right)^{T}$ and $b(\theta)=\theta_{1}^{2}+\theta_{2}^{2}-2$. Let us select the truncation degree $k=5$, by using the Taylor expansion of $\zeta_{i}$ one has

$$
\left\{\begin{aligned}
\dot{x}_{1}= & \frac{-x_{1}-5 x_{2}^{5}-x_{1}^{2}\left(\theta_{1}+\theta_{2}\right)}{1+x_{2}^{2}} \\
& -x_{2}\left(x_{1}-\frac{x_{1}^{3}}{3 !}+\frac{x_{1}^{5}}{5 !}+\xi_{1} \frac{x_{1}^{6}}{6 !}\right) \\
\dot{x}_{2}= & -2 x_{2} \theta_{2}-2 x_{1}^{3} \\
& -\frac{1}{\theta_{1}}\left(x_{2}+\frac{x_{2}^{2}}{2 !}+\frac{x_{2}^{3}}{3 !}+\frac{x_{2}^{4}}{4 !}+\frac{x_{2}^{5}}{5 !}+\xi_{2} \frac{x_{2}^{6}}{6 !}\right)
\end{aligned}\right.
$$

where the ranges of $\zeta_{1}$ and $\zeta_{2}$ can be obtained according to the selected Lyapunov function and its sublevel set, which 
will be shown in Example 2.

The more general case with non-polynomial parameterdependent functions $\zeta_{i}(x, \theta)$ is discussed in Section III.

\section{B. Problem Formulation}

In this paper, we aim to compute the sublevel set of a rational Lyapunov function to under-approximate $\mathcal{R}$. Specifically, let $v(x)$ be a rational function of system (1):

$$
v(x)=\frac{v_{\text {num }}(x)}{v_{\text {den }}(x)}
$$

where $v_{\text {num }} \in \mathcal{P}$ and $v_{\text {den }} \in \mathcal{P}$ fulfill

$$
\begin{gathered}
\forall x \in \mathcal{D}, \lim _{\|x\| \rightarrow \infty} v(x)=\infty, \\
\forall x \in \mathcal{D} /\left\{0_{n}\right\}, v_{\text {num }}(x)>0, \text { and } v_{\text {num }}\left(0_{n}\right)=0, \\
\forall x \in \mathcal{D}, v_{\text {den }}(x)>0,
\end{gathered}
$$

and $\mathcal{D}$ is defined in (1). The sublevel set of $v(x)$ is

$$
\mathcal{V}(c)=\left\{x \in \mathbb{R}^{n}: v(x) \leq c\right\}
$$

where $c \in \mathbb{R}$. The function $v(x)$ is a Lyapunov function of system (1) for the origin if

$$
\dot{v}(x, \theta)<0, \forall x \in \mathcal{D} /\left\{0_{n}\right\}, \forall \theta \in \Theta .
$$

We can now formally formulate our main problem: compute the largest under-estimate of the RDA using the sublevel set of a rational Lyapunov function $v(x)$, i.e., solving

$$
\begin{aligned}
& \mu^{*}=\sup _{c, v} c \\
& \text { s.t. }\left\{\begin{array}{l}
(11)-(14) \text { hold } \\
\mathcal{V}(c) \subseteq \mathcal{D} \\
\forall \theta \in \Theta, \forall \xi_{i} \in \Xi, \forall i=1, \ldots, r
\end{array}\right.
\end{aligned}
$$

where $\theta$ and $\Theta$ are introduced in (1) and (4), $\xi_{i}$ and $\Xi$ are introduced in (5) and (7).

\section{UNDER-APPROXIMATING THE RDA}

In this section, we will first give an estimation of the RDA by using a selected rational Lyapunov function. Then, the problem of the largest estimate of the RDA can be converted from a non-convex problem to a quasi-convex optimization problem. Based on this, a necessary and sufficient condition is proposed for establishing the tightness of this estimate.

\section{A. Estimation with Pre-defined Shape}

First, the system (1) can be rewritten in a compact parameter-dependent rational polynomial form as follows:

$$
\exists \xi \in \Xi, \dot{x}=h_{\mathrm{num}}(x, \tilde{\theta}) \oslash h_{\mathrm{den}}(x, \tilde{\theta})=h(x, \tilde{\theta})
$$

where $\tilde{\theta}=\left(\theta^{T}, \xi^{T}\right)^{T} \in \mathbb{R}^{n_{\tilde{\theta}}}, n_{\tilde{\theta}}=n_{\theta}+r, h_{\text {num }} \in \mathcal{P}^{n}$ and $h_{\text {den }} \in \mathcal{P}^{n}$ which can be obtained from $f, g_{i}$ and the Taylor expansion of $\zeta_{i}$ with a selected truncation degree $k$. Considering (4) and (7), a new constraint set for $\tilde{\theta}$ can be defined as follows:

$$
\widetilde{\Theta}=\left\{\tilde{\theta} \in \mathbb{R}^{n_{\tilde{\theta}}}: \tilde{a}(\tilde{\theta}) \geq 0, b(\theta)=0\right\}
$$

where $\tilde{a}(\tilde{\theta})=\left(a(\theta)^{T}, \xi_{1}-\underline{\tau}_{1}, \bar{\tau}_{1}-\xi_{1}, \ldots, \xi_{r}-\underline{\tau}_{r}, \bar{\tau}_{r}-\right.$ $\left.\xi_{r}\right)^{T} \in \mathbb{R}^{n_{\tilde{a}}}$ and $\bar{\tau}_{i}, \underline{\tau}_{i}$ are chosen according to (8). The following example is provided for illustration.
Example 2: Continued from Example 1, (10) can be equivalently expressed as

$$
\left\{\begin{aligned}
\dot{x}_{1} & =\frac{1}{-720\left(1+x_{2}^{2}\right)}\left(720 x_{1}+3600 x_{2}^{5}+720 x_{1}^{2} \theta_{1}\right. \\
& \left.+720 x_{1}^{2} \theta_{2}+720 x_{1} x_{2}-120 x_{1}^{3} x_{2}+6 x_{1}^{5} x_{2}+\xi_{1} x_{1}^{6} x_{2}\right) \\
\dot{x}_{2} & =\frac{1}{-720 \theta_{1}}\left(1440 x_{2} \theta_{1} \theta_{2}+1440 x_{1}^{3} \theta_{1}+720 x_{2}+360 x_{2}^{2}\right. \\
& \left.+120 x_{2}^{3}+30 x_{2}^{4}+6 x_{2}^{5}+\xi_{2} x_{2}^{6}\right)
\end{aligned}\right.
$$

with $a(\theta)=\left(\theta_{1}-1,2-\theta_{1}, \theta_{2}, 1-\theta_{2}\right)^{T}$ and $b(\theta)=\theta_{1}^{2}+\theta_{2}^{2}-2$. Let us consider a simple Lyapunov function $v_{\text {num }}=x_{1}^{2}+x_{2}^{2}$ and $v_{\text {den }}=1$. The bounds $\underline{\tau}_{i}$ and $\bar{\tau}_{i}$ in (8) can be selected as

$$
\begin{aligned}
& \underline{\tau}_{1}=-\sigma, \bar{\tau}_{1}=\sigma, \sigma= \begin{cases}\sin \sqrt{c}, & \text { if } \sqrt{c} \leq \pi / 2, \\
1, & \text { otherwise, }\end{cases} \\
& \underline{\tau}_{2}=-e^{\sqrt{c}}, \bar{\tau}_{2}=-e^{-\sqrt{c}},
\end{aligned}
$$

from which we have that $\tilde{a}(\tilde{\theta})=\left(\theta_{1}-1,2-\theta_{1}, \theta_{2}, 1-\theta_{2}, \xi_{1}-\right.$ $\left.\underline{\tau}_{1}, \bar{\tau}_{1}-\xi_{1}, \xi_{2}-\underline{\tau}_{2}, \bar{\tau}_{2}-\xi_{2}\right)^{T}$ and $b(\theta)=\theta_{1}^{2}+\theta_{2}^{2}-2$. The largest under-estimate of the RDA is shown in Fig. 1.

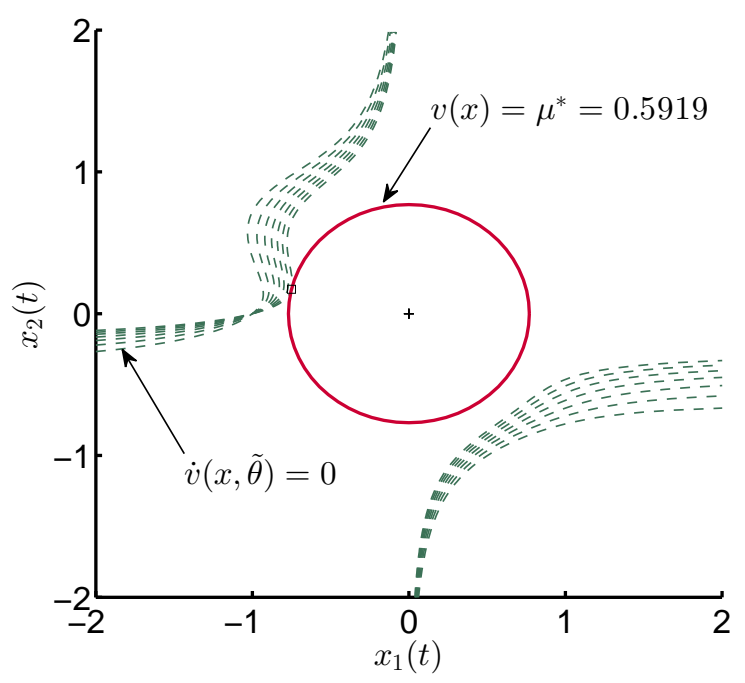

Fig. 1. Example 2: The solid red line indicates the bound of the largest estimate of the RDA by using $v(x)=x_{1}^{2}+x_{2}^{2}$; the dashed lines indicate $\dot{v}(x, \tilde{\theta})=0$ with $k=5$ for some $\tilde{\theta} \in \tilde{\Theta}$.

In order to check the nonnegativity over a semialgebraic set, real Positivestellensatz (P-satz) is verified to be a powerful tool [24]. The following result provides a stronger version of the P-satz:

Lemma 1: ([25]) $f_{1}, \ldots, f_{l}$ are polynomials of even degree such that the set

$$
\mathcal{F}=\left\{x \in \mathbb{R}^{n}: f_{1}(x) \geq 0, \ldots, f_{l}(x) \geq 0\right\}
$$

is compact and there are no common zeros for the highest degree forms in $\mathbb{R}_{0}^{n}$, then there exists a polynomial $p$ satifying

$$
\begin{aligned}
p(x)>0, \forall x \in \mathcal{F} \Longleftrightarrow & \exists s_{0}(x), \ldots, s_{l}(x) \in \mathcal{P}^{\mathrm{SOS}}: \\
& p(x)=s_{0}(x)+\sum_{i=1}^{l} s_{i}(x) f_{i}(x) .
\end{aligned}
$$


The above lemma points out that the cone generated by $f_{i}$ includes any strictly positive polynomial $p(x) \in \mathcal{F}$ over a semialgebraic set, which paves the way to estimate the RDA via SOS programming. Based on the above result, a new class of parameter-dependent polynomials is defined and the positivity of a parameter-dependent polynomial on a compact set can be established by exploiting the local SOS cone.

Definition 1 (Locally parameter-dependent SOS: $\mathcal{P}_{\mathrm{L}}^{\mathrm{SOS}}$ ): Consider a polynomial $p(x, \theta) \in \mathcal{P}, p(x, \theta)$ is an $\operatorname{SOS}$ polynomial on $\theta$ and a local SOS polynomial on $x$, i.e., $p(x, \theta)$ can be expressed as $p(x, \theta)=\sum_{i=1}^{m} p_{i}^{2}(x, \theta)$ for some polynomials $p_{i}(x, \theta)$ which are without monomials of degree 0 and 1 on $x$, then $p(x, \theta)$ is called a locally parameter-dependent SOS, denoted as $p \in \mathcal{P}_{\mathrm{L}}^{\mathrm{SOS}}$.

Lemma 2: For variable $x \in \mathbb{R}^{n}, \tilde{\theta} \in \mathbb{R}^{n_{\tilde{\theta}}}$, polynomials $p$, $a_{1}, \ldots, a_{m}$ and $b_{1}, \ldots, b_{l}$ depending both on $x$ and $\tilde{\theta}$, define the set

$$
\begin{aligned}
\mathcal{B}= & \left\{\left(x^{T}, \tilde{\theta}^{T}\right)^{T} \in \mathbb{R}^{n+n_{\tilde{\theta}}}: a_{i}(x, \tilde{\theta}) \geq 0,\right. \\
& \left.\forall i=1, \ldots, m, b_{j}(x, \tilde{\theta})=0, \forall j=1, \ldots, l\right\} .
\end{aligned}
$$

Let $\mathcal{B}$ be compact. Condition $\forall x \in \mathcal{B}: p(x, \tilde{\theta})>0$ can be established if the following condition holds:

$$
\left\{\begin{array}{l}
\exists s_{1}, \ldots, s_{m} \in \mathcal{P}_{\mathrm{L}}^{\mathrm{SOS}}, r_{1}, \ldots, r_{l} \in \mathcal{P} \\
p-\sum_{i=1}^{m} s_{i} a_{i}-\sum_{j=1}^{l} r_{i} b_{j} \in \mathcal{P}_{\mathrm{L}}^{\mathrm{SOS}} .
\end{array}\right.
$$

Proof: Considering (19) with $s_{0} \in \mathcal{P}_{\mathrm{L}}^{\mathrm{SOS}}$, this result can be obtained from Lemma 1 by expanding the dimension of state space from $n$ to $n+n_{\tilde{\theta}}$ and setting

$$
p=\sum_{i=1}^{m} s_{i} a_{i}+\sum_{j=1}^{l} r_{j} b_{j}+s_{0} .
$$

Remark 3: By exploiting the locally parameter-dependent SOS cone, condition (21) gives a sufficient condition to establish the positivity of a parameter-dependent polynomial on a compact set. Condition (21) is also a necessary condition if the degree of auxiliary locally parameter-dependent SOS polynomials $s_{i}$ is unlimited and there is a polynomial $b$ in set $\mathcal{B}$ such that $b^{-1}[0, \infty)$ is compact. For details please refer to [25] for the case of SOS cones.

The following result answers the question whether a sublevel set of a rational function is an estimate of the RDA.

Theorem 1: For a selected truncation degree $k$, consider a positive scalar $c \in \mathbb{R}^{+}$and a rational function $v(x)$ : $\mathbb{R}^{n} \rightarrow \mathbb{R}$ fulfilling (11)-(12), provided that there exist a polynomial $q(x, \tilde{\theta}): \mathbb{R}^{n+n_{\tilde{\theta}}} \rightarrow \mathbb{R}$ and polynomial vector $s(x, \tilde{\theta}): \mathbb{R}^{n+n_{\tilde{\theta}}} \rightarrow \mathbb{R}^{n_{\tilde{a}}}, r(x, \tilde{\theta}): \mathbb{R}^{n+n_{\tilde{\theta}}} \rightarrow \mathbb{R}^{n_{b}}$, such that

$$
\forall x \in \mathbb{R}_{0}^{n}, \forall \tilde{\theta} \in \mathbb{R}^{n_{\tilde{\theta}}}:\left\{\begin{array}{l}
\psi(x, \tilde{\theta}) \in \mathcal{P}_{\mathrm{L}}^{\mathrm{SOS}} \\
q(x, \tilde{\theta}) \in \mathcal{P}_{\mathrm{L}}^{\mathrm{SOS}} \\
s_{i} \in \mathcal{P}_{\mathrm{L}}^{\mathrm{SOS}}, i=1, \ldots, n_{\tilde{a}}
\end{array}\right.
$$

where

$$
\begin{aligned}
\psi(x, \tilde{\theta})= & w(x, \tilde{\theta})-q(x, \tilde{\theta})\left(c v_{\operatorname{den}}(x)-v_{\text {num }}\right) \\
& -s(x, \tilde{\theta})^{T} \tilde{a}(\tilde{\theta})-r(x, \tilde{\theta})^{T} b(\tilde{\theta}),
\end{aligned}
$$

and

$$
\begin{aligned}
w(x, \tilde{\theta})= & \left(v_{\text {num }}(x) \nabla v_{\operatorname{den}}(x)-v_{\operatorname{den}}(x) \nabla v_{\text {num }}(x)\right)^{T} \\
& \cdot\left(\left(h_{\text {num }}(x, \tilde{\theta}) \circ \eta(x, \tilde{\theta})\right) \cdot \operatorname{lcm}\left(\mathrm{h}_{\operatorname{den}}(\mathrm{x}, \tilde{\theta})\right)\right),
\end{aligned}
$$

functions $v_{\text {num }}$ and $v_{\text {den }}$ are introduced by (11), functions $h_{\text {num }}$ and $h_{\text {den }}$ are introduced by (16), operation $\nabla(\cdot)$ gives the gradient of a function, operation $1 \mathrm{~cm}$ gives the least common multiple of all the entries of a vector, operation $\circ$ is the element-wise product, operation $\oslash$ is the element-wise division and $\eta$ is a vector polynomial function

$$
\eta(x, \tilde{\theta})=\left(1_{n} \oslash h_{\operatorname{den}}(x, \tilde{\theta})\right) \cdot \operatorname{lcm}\left(\mathrm{h}_{\mathrm{den}}(\mathrm{x}, \tilde{\theta})\right)
$$

where $1_{n}$ is the ones vector with the dimension $n$ and all the elements being 1 . Then, $v(x)$ is a common Lyapunov function for the origin, and $\mathcal{V}(c) \subseteq \mathcal{R}$.

Proof: Our main effort in this proof is to derive that the rational function $v(x)$ is a common Lyapunov function of system (16) given (23), then it yields that $\mathcal{V}(c) \subseteq \mathcal{R}$ [1].

Suppose that (23) holds, since $c v_{\text {den }}(x)-v_{\text {num }} \geq 0$, $\tilde{a}(\tilde{\theta}) \geq 0, b(\tilde{\theta})=0, q(x, \tilde{\theta}) \in \mathcal{P}_{\mathrm{L}}^{\mathrm{SOS}}$ and $s_{i}(x, \tilde{\theta}) \in \mathcal{P}_{\mathrm{L}}^{\mathrm{SOS}}$, for $i=1, \ldots, n_{\tilde{a}}$, from Lemma 2 , one has that

$$
\forall x \in \mathcal{V}(c) /\left\{0_{n}\right\}, \forall \tilde{\theta} \in \widetilde{\Theta}: w(x, \tilde{\theta})>0 .
$$

Based on this, by using (25) and (26), it yields that

$$
\begin{aligned}
& 0<w(x, \tilde{\theta}) \\
& 0<\frac{w(x, \tilde{\theta})}{v_{\operatorname{den}}(x)^{2} \cdot \operatorname{lcm}\left(\mathrm{h}_{\operatorname{den}}(\mathrm{x}, \tilde{\theta})\right)^{2}} \\
& 0<\left(\frac{v_{\text {num }}(x) \nabla v_{\text {den }}(x)-v_{\text {den }}(x) \nabla v_{\text {num }}(x)}{v_{\text {den }}(x)^{2}}\right)^{T} \\
& \cdot\left(\frac{h_{\mathrm{num}}(x, \tilde{\theta}) \circ \eta(x, \tilde{\theta})}{\operatorname{lcm}\left(\mathrm{h}_{\mathrm{den}}(\mathrm{x}, \tilde{\theta})\right)}\right) \\
& 0<\left(\frac{v_{\text {num }}(x) \nabla v_{\text {den }}(x)-v_{\text {den }}(x) \nabla v_{\text {num }}(x)}{v_{\text {den }}(x)^{2}}\right)^{T} \\
& \text { - } h_{\text {num }}(x, \tilde{\theta}) \circ\left(1_{n} \oslash h_{\operatorname{den}}(x, \tilde{\theta})\right) \text {. }
\end{aligned}
$$

In addition, from (11) and (16), one has that there exists a $\xi \in \Xi$ (thus a $\tilde{\theta} \in \tilde{\Theta}$ ) such that

$$
\begin{array}{r}
\dot{v}(x, \tilde{\theta})=\left(\frac{v_{\mathrm{den}}(x) \nabla v_{\text {num }}(x)-v_{\text {num }}(x) \nabla v_{\operatorname{den}}(x)}{v_{\operatorname{den}}(x)^{2}}\right)^{T} \\
\cdot\left(h_{\text {num }}(x, \tilde{\theta}) \oslash h_{\operatorname{den}}(x, \tilde{\theta})\right) .
\end{array}
$$

Meanwhile, considering the fact that

$$
\begin{aligned}
& \left(h_{\mathrm{num}}(x, \tilde{\theta}) \oslash h_{\mathrm{den}}(x, \tilde{\theta})\right)= \\
& h_{\text {num }}(x, \tilde{\theta}) \circ\left(1_{n} \oslash h_{\mathrm{den}}(x, \tilde{\theta})\right),
\end{aligned}
$$

and comparing (28) with (29), it yields that from (28)

$$
\forall \tilde{\theta} \in \tilde{\Theta}: 0<-\dot{v}(x, \tilde{\theta}) .
$$

Therefore, one has that $v(x)$ is a Lyapunov function for the origin and $\mathcal{V}(c) \subseteq \mathcal{R}$ which completes this proof.

Remark 4: For this theorem, it is worth noting that 
- The conservatism of this approach stems from the bounded degrees of the auxiliary functions $q, s$ and $r$ (see Remark 3). Another source of conservatism is that, for system (1), the rational Lyapunov function is usually not the maximal Lyapunov function by which the exact DA can be obtained. In other words, the rational Lyapunov function can only be used to approximate the maximal Lyapunov function, making this method an under-estimation of the exact RDA.

- This method can also be extended to a more general case with multi-variable parameter-dependent nonpolynomial functions $\zeta_{i}(x, \theta)$ in system (1), by using the method of multi-variable truncated Taylor expansion. However, this extension may result in a large number of parameters $\xi_{i}$ for expressing the Taylor remainder, which is computationally demanding.

\section{B. Selection of the Lyapunov function}

In this subsection, a simple strategy for choosing an initial Lyapunov function will be introduced. In particular, let us introduce the Jacobian matrix

$$
A(\tilde{\theta})=\left.\frac{d h(x, \tilde{\theta})}{d x}\right|_{x=0_{n}}
$$

and a symmetric matrix $P$ such that

$$
\forall \tilde{\theta} \in \widetilde{\Theta},\left\{\begin{array}{l}
P>0 \\
P A(\tilde{\theta})+A(\tilde{\theta}) P<0 .
\end{array}\right.
$$

Observe that the condition (31) is equivalent to the condition that there exists a quadratic Lyapunov function for the linearized system, which can be established by using the existing SOS matrix techniques.

One way to construct the rational Lyapunov function is

$$
v(x)=\frac{v_{\mathrm{q}}+v_{\mathrm{a}}}{v_{\mathrm{den}}}
$$

fulfilling (12) where

$$
v_{\mathrm{q}}(x)=x^{T} P x
$$

is the quadratic Lyapunov function for the origin, with $P$ satisfying (31), and $v_{\mathrm{a}}$ is an auxiliary polynomial function which can be simply chosen as $\left(x^{T} x\right) \cdot\left(x^{T} P x\right)$. In fact, $v_{\text {a }}$ can be selected as any polynomial such that (11)-(12) hold and

$$
\left.\nabla v_{\mathrm{a}}(x)\right|_{x=0_{n}}=0,\left.\nabla^{2} v_{\mathrm{a}}(x)\right|_{x=0_{n}}=0 .
$$

More details for choosing the initial rational Lyapunov function, please refer to [26].

It is useful to mention that the search for an optimal rational Lyapunov function to enlarge the RDA may provide a less conservative result, but it is out of the scope of this paper due to limited space. The readers may refer to [5], [23] for the case of polynomial Lyapunov functions.

\section{Square Matrix Representation (SMR) based Quasi- Convex Optimization}

Notice that the condition of Theorem 1 is not easy to establish because it turns out to be a non-convex problem for finding a locally parameter-dependent $\operatorname{SOS} s(x, \tilde{\theta})$ and a positive scalar $c$ at the same time. In addition, another challenge is that there is no existing method for locally parameter-dependent SOS programming. In order to solve these issues, a new class of SMR is proposed for the set of locally parameter-dependent $\mathrm{SOS}$, i.e., $p(x, \tilde{\theta}) \in \mathcal{P}_{\mathrm{L}}^{\mathrm{SOS}}$, and an approach is provided to convert this non-convex problem to a quasi-convex optimization problem.

In the following, we will first recall the SMR method and then introduce the class of SMR for the set $\mathcal{P}_{\mathrm{L}}^{\mathrm{SOS}}$. Consider a polynomial $p_{1}(x) \in \mathcal{P}$ of degree $\operatorname{deg}_{x}\left(p_{1}\right)$, define $d_{x}^{p_{1}}$ as the smallest integer not less than $\frac{\operatorname{deg}_{x}\left(p_{1}\right)}{2}$, i.e., $d_{x}^{p_{1}}=\left\lceil\frac{\operatorname{deg}_{x}\left(p_{1}\right)}{2}\right\rceil$, we can express $p_{1}(x)$ in SMR as:

$$
p_{1}(x)=(*)^{T}\left(P_{1}+L_{1}(\delta)\right) \phi_{\mathrm{p}}\left(n, d_{x}^{p_{1}}\right)
$$

where $(*)^{T} A B$ is short for $B^{T} A B$ introduced in Section II, $P_{1}$ is denoted by the SMR matrix of $p_{1}(x), n$ is the number of variables, $\phi_{\mathrm{p}}\left(n, d_{x}^{p_{1}}\right) \in \mathbb{R}^{l_{1}}$ is called the power vector containing all monomials of degree less or equal to $d_{x}^{p_{1}}, L_{1}(\delta)$ is a parameterization of the affine space

$$
\begin{aligned}
\mathscr{L}_{1}= & \left\{L_{1}(\delta) \in \mathbb{R}^{l_{1} \times l_{1}}: L_{1}(\delta)=L_{1}^{T}(\delta),\right. \\
& \left.(*)^{T} L_{1}(\delta) \phi_{\mathrm{p}}\left(n, d_{x}^{p_{1}}\right)=0\right\},
\end{aligned}
$$

in which $\delta$ is a vector of free parameters. An exemple of SMR is provided below.

Example 3: Given a polynomial $p_{1}(x)=7-3 x+6 x^{2}+$ $4 x^{3}+5 x^{4}$, we have $d_{x}^{p_{1}}=2, n=1$ and $\phi_{\mathrm{p}}\left(n, d_{x}^{p_{1}}\right)=$ $\left(1, x, x^{2}\right)^{T}$. Then, $p_{1}(x)$ can be expressed using (35) with

$$
P_{1}=\left(\begin{array}{ccc}
7 & -1.5 & 0 \\
-1.5 & 6 & 2 \\
0 & 2 & 5
\end{array}\right), L_{1}(\delta)=\left(\begin{array}{ccc}
0 & 0 & \delta \\
0 & -2 \delta & 0 \\
\delta & 0 & 0
\end{array}\right) \text {. }
$$

Now, let us consider a locally parameter-dependent polynomial $p_{2}(x, \tilde{\theta}) \in \mathcal{P}_{\mathrm{L}}^{\mathrm{SOS}}$ with degree $\operatorname{deg}_{x}$ on $x$ and with degree $\operatorname{deg}_{\tilde{\theta}}$ on $\tilde{\theta}$, and $\tilde{\theta} \in \mathbb{R}^{n_{\tilde{\theta}}}$. Thus, $p_{2}(x, \tilde{\theta})$ can be expressed in the SMR form as

$$
p_{2}(x, \tilde{\theta})=(*)^{T}\left(P_{2}+L_{2}(\delta)\right)\left(\phi_{1}\left(n, d_{x}^{p_{2}}\right) \otimes \phi_{\mathrm{p}}\left(n_{\tilde{\theta}}, d_{\tilde{\theta}}^{p_{2}}\right)\right)
$$

where $d_{x}^{p_{2}}=\left\lceil\frac{\operatorname{deg}_{x}\left(p_{2}\right)}{2}\right\rceil, d_{\tilde{\theta}}^{p_{2}}=\left\lceil\frac{\operatorname{deg}_{\tilde{\theta}}\left(p_{2}\right)}{2}\right\rceil, \phi_{1}\left(n, d_{x}^{p_{2}}\right) \in \mathbb{R}^{l_{2}}$ is a power vector containing all the monomials of degree less or equal to $d_{x}^{p_{2}}$ except degrees 1 and $0 . L_{2}(\delta)$ is a parameterization of the affine space

$$
\begin{aligned}
\mathscr{L}_{2}= & \left\{L_{2}(\alpha) \in \mathbb{R}^{l_{3} \times l_{3}}: L_{2}(\alpha)=L_{2}^{T}(\alpha),\right. \\
& \left.(*)^{T} L_{1}(\alpha)\left(\phi_{1}\left(n, d_{x}^{p_{2}}\right) \otimes \phi_{\mathrm{p}}\left(n_{\tilde{\theta}}, d_{\tilde{\theta}}^{p_{2}}\right)\right)=0\right\}
\end{aligned}
$$

in which $\alpha$ is a vector of free parameters, $l_{3}=l_{2} \cdot l_{1}$. Let us introduce

$$
\begin{aligned}
q(x, \tilde{\theta})= & (*)^{T} Q\left(\phi_{\mathrm{l}}\left(n, d_{x}^{q}\right) \otimes \phi_{\mathrm{p}}\left(n_{\tilde{\theta}}, d_{\tilde{\theta}}^{q}\right)\right) \\
s_{i}(x, \tilde{\theta})= & (*)^{T} S_{i}\left(\phi_{\mathrm{p}}\left(n, d_{x}^{s_{i}}\right) \otimes \phi_{\mathrm{l}}\left(n_{\tilde{\theta}}, d_{\tilde{\theta}}^{s_{i}}\right)\right) \\
& \forall i=1, \ldots, n_{\tilde{a}} \\
r_{i}(x, \tilde{\theta})= & R_{i}^{T}\left(\phi_{\mathrm{l}}\left(n, \operatorname{deg}_{x}\left(r_{i}\right)\right) \otimes \phi_{\mathrm{p}}\left(n_{\tilde{\theta}}, \operatorname{deg}_{\tilde{\theta}}\left(r_{i}\right)\right)\right) \\
& \forall i=1, \ldots, n_{b} .
\end{aligned}
$$


Based on these expressions, we further define

$$
\begin{aligned}
\psi(x, \tilde{\theta})= & (*)^{T}(\Psi(c, Q, S, R)+L(\alpha)) \\
& \cdot\left(\phi_{\mathrm{l}}\left(n, d_{x}^{\psi}\right) \otimes \phi_{\mathrm{p}}\left(n_{\tilde{\theta}}, d_{\tilde{\theta}}^{\psi}\right)\right) \\
S= & \operatorname{diag}\left(S_{1}, \ldots, S_{n_{\tilde{a}}}\right) \\
R= & \left(R_{1}^{T}, \ldots, R_{n_{b}}^{T}\right)^{T} .
\end{aligned}
$$

These expressions of SMR lead to a result for estimating the largest RDA as follows:

Lemma 3: For a selected truncation degree $k$, consider a rational function $v(x): \mathbb{R}^{n} \rightarrow \mathbb{R}$ satisfying (11)-(12), suppose that there exist $Q, S, R$ and $\alpha$ defined in (39)-(40) such that

$$
\begin{aligned}
& \mu_{k}=\sup _{c, Q, S, R, \alpha} c \\
& \text { s.t. }\left\{\begin{array}{l}
\Psi(c, Q, S, R)+L(\alpha)>0 \\
Q>0 \\
S>0 .
\end{array}\right.
\end{aligned}
$$

Then, $\mu_{k}$ is a lower bound of $\mu^{*}$, i.e., $\mu_{k} \leq \mu^{*}$.

Proof: Suppose that condition (41) holds. By premultiplying and post-multiplying the first inequality of (41) by $\left(\phi_{\mathrm{l}}\left(n, d_{x}\right) \otimes \phi_{\mathrm{p}}\left(n_{\tilde{\theta}}, d_{\tilde{\theta}}\right)\right)^{T}$ and $\phi_{\mathrm{l}}\left(n, d_{x}\right) \otimes \phi_{\mathrm{p}}\left(n_{\tilde{\theta}}, d_{\tilde{\theta}}\right)$, respectively, one has that $\psi(x, \tilde{\theta})>0$ and $\Psi(c, Q, S, R)+L(\alpha)$ is the SMR matrix of $\psi(x, \tilde{\theta})>0$ based on the first equation of (40). In addition, considering the fact that $\phi_{1}\left(n, d_{x}\right) \neq 0$ holds when $x \neq 0$, one has that $\psi(x, \tilde{\theta}) \in \mathcal{P}_{\mathrm{L}}^{\mathrm{SOS}}$ for all $x \in \mathbb{R}_{0}^{n}$ and for all $\tilde{\theta} \in \mathbb{R}^{n_{\tilde{\theta}}}$, based on Definition 1 . In the same way, one obtains that $q(x, \tilde{\theta}) \in \mathcal{P}_{\mathrm{L}}^{\mathrm{SOS}}$ and $s_{i}(x, \tilde{\theta}) \in \mathcal{P}_{\mathrm{L}}^{\mathrm{SOS}}$ for all $x \in \mathbb{R}^{n}$, for all $\tilde{\theta} \in \mathbb{R}^{n_{\tilde{\theta}}}$, for all $i=1, \ldots, n_{\tilde{a}}$. Then, the condition (23) holds. Therefore, $\mathcal{V}(c)$ is an estimate of the RDA with the truncation degree $k$. Taking into account the definition of $\mu^{*}$ in (15), it implies that $\mu_{k}$ is a lower bound of $\mu^{*}$ which ends this proof.

Let us observe that the optimization (41) is a problem of bilinear matrix inequalities owing to the product of $Q$ and $c$. One possible way to solve this problem is to use a bisection algorithm on $c$ where an LMI feasibility test is solved for every fixed value of $c$ [11]. However, this method may lead to a great number of LMI feasibility tests, which is computationally demanding.

For this reason, we propose an approach of quasi-convex optimization, and a generalized eigenvalue problem (GEVP) is constructed in order to solve (41). First, let us introduce the following transformation based on which the GEVP can be properly formulated.

Lemma 4: Define the polynomials

$$
\begin{aligned}
\tilde{v}(x)= & v_{\mathrm{den}}(x)+\lambda v_{\text {num }} \\
u(x, \tilde{\theta})= & u_{1}(x, \tilde{\theta})+u_{2}(x, \tilde{\theta}) \\
u_{1}(x, \tilde{\theta})= & w(x, \tilde{\theta})+q(x, \tilde{\theta}) v_{\text {num }}(x) \\
& -s(x, \tilde{\theta})^{T} \tilde{a}(\tilde{\theta})-r(x, \tilde{\theta})^{T} b(\tilde{\theta}) \\
u_{2}(x, \tilde{\theta})= & q(x, \tilde{\theta}) \tilde{v}(x)
\end{aligned}
$$

and let $U_{2}$ be the SMR matrix of $u_{2}$ with the power vector $\phi_{\mathrm{l}}\left(n, d_{x}^{u}\right) \otimes \phi_{\mathrm{p}}\left(n_{\tilde{\theta}}, d_{\tilde{\theta}}^{u}\right), \widetilde{V}$ be the SMR matrix of $\tilde{v}$ with the power vector $\phi_{1}\left(n, d_{x}^{\tilde{v}}\right)$. Then, $U_{2}$ can be expressed by

$$
U_{2}=(*)^{T}(Q \otimes \tilde{V}) H
$$

where $\phi_{1}\left(n, d_{x}^{q}\right) \otimes \phi_{\mathrm{p}}\left(n_{\tilde{\theta}}, d_{\tilde{\theta}}^{q}\right) \otimes \phi_{\mathrm{l}}\left(n, d_{x}^{\tilde{v}}\right)=H\left(\phi_{\mathrm{l}}\left(n, d_{x}^{u}\right) \otimes\right.$ $\left.\phi_{\mathrm{p}}\left(n_{\tilde{\theta}}, d_{\tilde{\theta}}^{u}\right)\right)$.

Proof: By exploiting the Kronecker product [27] and its mixed-product property:

$$
(A \otimes B)(C \otimes D)=(A C) \otimes(B D),
$$

the product of $q$ and $\tilde{v}$ can be expressed in the SMR form by a common power vector. Specifically,

$$
\begin{aligned}
q(x, \tilde{\theta}) \tilde{v}(x)= & (*)^{T} Q\left(\phi_{\mathrm{l}}\left(n, d_{x}^{q}\right) \otimes \phi_{\mathrm{p}}\left(n_{\tilde{\theta}}, d_{\tilde{\theta}}^{q}\right)\right) \\
& \cdot(*)^{T} \widetilde{V}\left(\phi_{\mathrm{l}}\left(n, d_{x}^{\tilde{v}}\right)\right. \\
\stackrel{(47)}{=} & (*)^{T}(Q \otimes \widetilde{V}) \\
& \cdot\left(\phi_{\mathrm{l}}\left(n, d_{x}^{q}\right) \otimes \phi_{\mathrm{p}}\left(n_{\tilde{\theta}}, d_{\tilde{\theta}}^{q}\right) \otimes \phi_{\mathrm{l}}\left(n, d_{x}^{\tilde{v}}\right)\right) \\
= & (*)^{T} H^{T}(Q \otimes \widetilde{V}) H \\
& \cdot\left(\phi_{\mathrm{l}}\left(n, d_{x}^{u}\right) \otimes \phi_{\mathrm{p}}\left(n_{\tilde{\theta}}, d_{\tilde{\theta}}^{u}\right)\right) \\
= & (*)^{T} U_{2}\left(\phi_{\mathrm{l}}\left(n, d_{x}^{u}\right) \otimes \phi_{\mathrm{p}}\left(n_{\tilde{\theta}}, d_{\tilde{\theta}}^{u}\right)\right),
\end{aligned}
$$

by which we conclude this proof.

Theorem 2: For a selected truncation degree $k$, consider a positive scalar $\lambda \in \mathbb{R}^{+}$and a rational function $v(x): \mathbb{R}^{n} \rightarrow$ $\mathbb{R}$ satisfying (11)-(12), $\mu_{k}$ in (41) can be computed by

$$
\mu_{k}=-\frac{\tilde{e}}{1+\lambda \tilde{e}}
$$

where $\tilde{e}$ is the solution of the following GEVP

$$
\begin{aligned}
& \tilde{e}=\inf _{e, Q, R, \alpha} e \\
& \text { s.t. }\left\{\begin{array}{l}
Q>0 \\
S>0 \\
e U_{2}(Q)>-U_{1}(Q, R, S)-L(\alpha) .
\end{array}\right.
\end{aligned}
$$

Proof: From the last inequality of (49), one has

$$
e U_{2}(Q)+U_{1}(Q, R, S)+L(\alpha)>0,
$$

which is the SMR matrix of the polynomial $e u_{2}(x, \tilde{\theta})+$ $u_{1}(x, \tilde{\theta})$, and it can be expressed as

$$
\begin{array}{ll}
\text { (44),(45) } & e u_{2}(x, \tilde{\theta})+u_{1}(x, \tilde{\theta}) \\
& e q(x, \tilde{\theta}) \tilde{v}(x)+w(x, \tilde{\theta})+q(x, \tilde{\theta}) v_{\text {num }}(x) \\
& -s(x, \tilde{\theta})^{T} \tilde{a}(\tilde{\theta})-r(x, \tilde{\theta})^{T} b(\tilde{\theta}) \\
\stackrel{(42)}{=} \quad & q(x, \tilde{\theta})\left(e \lambda v_{\text {num }}(x)+e v_{\operatorname{den}}(x)+v_{\text {num }}(x)\right) \\
& +w(x, \tilde{\theta})-s(x, \tilde{\theta})^{T} \tilde{a}(\tilde{\theta})-r(x, \tilde{\theta})^{T} b(\tilde{\theta}) \\
=\quad & -(1+\lambda e)\left(\frac{-e}{1+\lambda e} v_{\operatorname{den}}(x)-v_{\text {num }}(x)\right) q(x, \tilde{\theta}) \\
& +w(x, \tilde{\theta})-s(x, \tilde{\theta})^{T} \tilde{a}(\tilde{\theta})-r(x, \tilde{\theta})^{T} b(\tilde{\theta}) .
\end{array}
$$

Notice that for all $e \in(-1 / \lambda, 0]$, the function $-e /(1+\lambda e)$ is monotonically decreasing and its corresponding mapping range is the interval $[0,+\infty)$. It directly yields that the lower bound of $\tilde{\gamma}$ can be calculated by (48).

Next, let us prove $U_{2}(Q)$ is positive definite which makes (49) a GEVP. Considering the dimension of matrices $U_{2}$, $Q$ and $\widetilde{V}$, one has that a shrunk SMR matrix is obtained after the power vector transformation in (46). Moreover, any monomial of the power vector $\phi_{\mathrm{l}}\left(n, d_{x}^{q}\right) \otimes \phi_{\mathrm{p}}\left(n_{\tilde{\theta}}, d_{\tilde{\theta}}^{q}\right) \otimes$ 
$\phi_{\mathrm{l}}\left(n, d_{x}^{\tilde{v}}\right)$ is included in the monomial set of the power vector $\left(\phi_{\mathrm{l}}\left(n, d_{x}^{u}\right) \otimes \phi_{\mathrm{p}}\left(n_{\tilde{\theta}}, d_{\tilde{\theta}}^{u}\right)\right)$, which directly implies that matrix $H$ has full rank. From Lemma 4 , one has $U_{2}>0$ if $Q>0$ and $\widetilde{V}>0$, which completes this proof.

Note that the last constraint in (49) is called the linearfractional LMI. For more details of the formulation of the GEVP, please refer to [6].

\section{Tightness Investigation}

The last theorem gives a useful strategy to compute a guaranteed lower bound of $\mu_{k}$ for a specific truncation degree $k$. Naturally one may ask: Is this lower bound tight? The following theorem is proposed to answer this question.

Theorem 3: Let $\bar{Q}, \bar{R}, \bar{S}$ and $\bar{\alpha}$ be the optimal values of $Q, R, S$ and $\alpha$ in the optimization (49), and define

$$
\bar{\Gamma}(\bar{Q}, \bar{R}, \bar{S}, \bar{\alpha})=\tilde{e} U_{2}(\bar{Q})+U_{1}(\bar{Q}, \bar{R}, \bar{S})+L(\bar{\alpha}) .
$$

Then, a necessary and sufficient condition for $\mu_{k}=\mu^{*}$ is that there exists a $(\bar{x}, \bar{\theta}) \in \mathcal{T}$ where

$$
\begin{aligned}
\mathcal{T}=\left\{(x, \tilde{\theta}) \in \mathbb{R}_{0}^{n} \times \mathbb{R}^{n_{t}}:\right. & \\
& \left.\phi_{\mathrm{l}}\left(n, d_{\bar{x}}^{u}\right) \otimes \phi_{\mathrm{p}}\left(n_{\tilde{\theta}}, d_{\bar{\theta}}^{u}\right) \in \operatorname{ker}(\bar{\Gamma}) \text { and } \dot{v}(\bar{x}, \bar{\theta})=0\right\} .
\end{aligned}
$$

Proof: (Sufficiency) Let us recall that the largest estimate of the RDA can be obtained by $\mathcal{V}(c)$ where

$$
\mu=\sup _{x \in \mathbb{R}_{0}^{n}, \tilde{\theta} \in \widetilde{\Theta}} v(x) \text { s.t. } \dot{v}(x, \tilde{\theta})=0 .
$$

Let the optimum of (53) be $\bar{x}$ and $\bar{\theta}$. From (25), one has

$$
w(\bar{x}, \bar{\theta})=0, v_{\text {num }}(\bar{x})-\mu v_{\operatorname{den}}(\bar{x})=0 .
$$

In addition, let us observe that

$$
\begin{aligned}
0 \underset{(50),(52)}{=} & (*)^{T} \bar{\Gamma}(\bar{Q}, \bar{R}, \bar{S}, \bar{\alpha})\left(\phi_{\mathrm{l}}\left(n, d_{\bar{x}}^{u}\right) \otimes \phi_{\mathrm{p}}\left(n_{\tilde{\theta}}, d_{\bar{\theta}}^{u}\right)\right) \\
\stackrel{(44),(45)}{=} & -(1+\lambda e)\left(\mu v_{\mathrm{den}}(\bar{x}, \bar{\theta})+v_{1}(\bar{x}, \bar{\theta})\right. \\
& \left.+w(\bar{x}, \bar{\theta})-s(\bar{x}, \tilde{\theta})^{T} \tilde{a}(\bar{\theta})-r(\bar{x}, \bar{\theta})\right) q(\bar{x}, \bar{\theta}) \\
&
\end{aligned}
$$

Since $\tilde{a}(\bar{\theta}) \geq 0$ and $b(\bar{\theta})=0$, it follows that

$$
\begin{array}{ll} 
& e u_{2}(\bar{x}, \bar{\theta})+u_{1}(\bar{x}, \bar{\theta}) \\
\leq \quad & -(1+\lambda e)\left(\mu v_{\operatorname{den}}(\bar{x})-v_{\text {num }}(\bar{x})\right) q(\bar{x}, \bar{\theta}) \\
& +w(\bar{x}, \bar{\theta}) \\
\stackrel{(54)}{=} & 0,
\end{array}
$$

which directly yields that $(*)^{T} \bar{\Gamma}(\bar{Q}, \bar{R}, \bar{S}, \bar{\alpha})\left(\phi_{\mathrm{l}}\left(n, d_{\bar{x}}^{u}\right) \otimes\right.$ $\left.\phi_{\mathrm{p}}\left(n_{\tilde{\theta}}, d_{\bar{\theta}}^{u}\right)\right)=0$. Taking into account that $\bar{\Gamma}$ is semidefinite, one has $\phi_{1}\left(n, d_{\bar{x}}^{u}\right) \otimes \phi_{\mathrm{p}}\left(n_{\tilde{\theta}}, d_{\bar{\theta}}^{u}\right)$ is in the null space of $\bar{\Gamma}$.

(Necessity) Let us suppose $\phi_{\mathrm{l}}\left(n, d_{\bar{x}}^{u}\right) \otimes \phi_{\mathrm{p}}\left(n_{\tilde{\theta}}, d_{\bar{\theta}}^{u}\right) \in$ $\operatorname{ker}(\bar{\Gamma})$, it follows that

$$
\begin{array}{cl}
0 \stackrel{(52),(51)}{=} & (*)^{T} \tilde{\Gamma}(\bar{Q}, \bar{R}, \bar{S}, \tilde{\alpha})\left(\phi_{\mathrm{l}}\left(n, d_{\bar{x}}^{u}\right) \otimes \phi_{\mathrm{p}}\left(n_{\tilde{\theta}}, d_{\bar{\theta}}^{u}\right)\right) \\
& -(1+\lambda e)\left(\frac{-e}{1+\lambda e} v_{\operatorname{den}}(\bar{x})-v_{\text {num }}(\bar{x})\right) q(\bar{x}, \bar{\theta}) \\
& +w(\bar{x}, \bar{\theta})-s(\bar{x}, \bar{\theta})^{T} \tilde{a}(\bar{\theta})-r(\bar{x}, \bar{\theta})^{T} b(\bar{\theta}) .
\end{array}
$$

Then, taking into account that $\dot{v}(\bar{x}, \bar{\theta})=0, \tilde{a}(\bar{\theta}) \geq 0$ and $b(\bar{\theta})=0$, one has $v(\bar{x})=\mu_{k}$. It further implies that $\mu^{*} \leq$ $v(\bar{x})=\mu_{k}$, while $\mu_{k}$ is a lower bound of $\mu^{*}$, i.e., $\mu_{k} \leq \mu^{*}$. Thus, one has $\mu^{*}=\mu_{k}$.
The above result gives a necessary and sufficient condition for verifying the tightness of the lower bound of $\mu^{*}$. This result is demonstrated by the following example.

\section{An ILlustrative EXAmple}

Computation is carried out using MATLAB R2014a on a standard laptop with a $2.3 \mathrm{GHz}$ Intel Core $17-4712 \mathrm{MQ}$ processor and an 8GB DDR3 RAM.

Consider a 2-dimensional non-polynomial system

$$
\left\{\begin{array}{l}
\dot{x}_{1}=\frac{x_{2}-\theta_{1}^{2} x_{1}}{2+x_{1}^{2}}-\theta_{2} x_{1}^{2}-5 x_{2}^{3}-\sin \left(x_{1}\right) \\
\dot{x}_{2}=1-\frac{2 \theta_{2} x_{2}-4 x_{1}^{3}}{1+x_{2}^{2}}-5 \theta_{1} x_{2}-e^{x_{2}}
\end{array}\right.
$$

with $g_{1}=g_{2}=1, \zeta_{1}=\sin \left(x_{1}\right), \zeta_{2}=1-e^{x_{2}}, \Theta=\{\theta \in$ $\left.\mathbb{R}^{2} \mid 0 \leq \theta_{1} \leq 1, \quad 0.5 \leq \theta_{2} \leq 2, \theta_{1}^{2}+\theta_{2}^{2} \leq 3\right\}$. Let us consider the estimation problem of the RDA by selecting the rational Lyapunov function via (32) as

$$
v_{1}(x)=\frac{x_{1}^{2}+x_{2}^{2}+x_{1}^{4}-x_{1}^{2} x_{2}^{2}+x_{2}^{4}}{2+x_{1}-2 x^{2}+2 x_{1}^{2}+4 x_{2}^{2}} .
$$

The lower bound $\mu_{k}$ can be obtained by solving the GEVP in (49). The result of computation is shown in Tab. I subject to different truncation degrees $k$. We compare this approach with the method of polynomial Lyapunov functions and choose a quadratic Lyapunov function $v_{2}(x)=x_{1}^{2}+x_{2}^{2}$ [28]. The result shows that, by contrast, both the unitary sublevel set $\mathcal{V}(1)$ and the largest estimate of the RDA are much smaller than the proposed approach by using $v_{1}(x)$ (see Fig. 2).

TABLE I

THE LOWER BOUND $\mu_{k}$ FOR SOME VALUES OF TRUNCATION DEGREE $k$ AND THE CORRESPONDING COMPUTATIONAL TIME $t_{c}$.

\begin{tabular}{cccccc}
\hline$k$ & 1 & 2 & 3 & 4 & 5 \\
\hline$\mu_{k}$ & 0.784 & 1.142 & 1.307 & 1.327 & 1.348 \\
$t_{c}[\mathrm{~s}]$ & 6.214 & 7.764 & 8.358 & 9.945 & 8.863 \\
\hline
\end{tabular}

Then, we would like to check whether the tightness of the lower bound is established. By using the condition (52) from Theorem 3, one obtains that the tightness is established with $\mu_{k}=\mu^{*}$ at point $\bar{x}=(-2.309,-2.863)^{T}$ and $\bar{\theta}=$ $(0.416,0.737,0.174,2.146)^{T}$ where $\bar{x} \in \mathcal{T}$.

\section{CONClusion And Future WORK}

For uncertain rational polynomial systems and nonpolynomial systems, a novel approach is proposed to compute the largest estimate of the RDA. A criterion based on SOS conditions is proposed for establishing whether a sublevel set of a rational function is in the RDA. Then, by introducing a new class of SMR for the set of locally parameter-dependent SOS and a transformation of power vector, the lower bound of the largest estimate of the RDA 


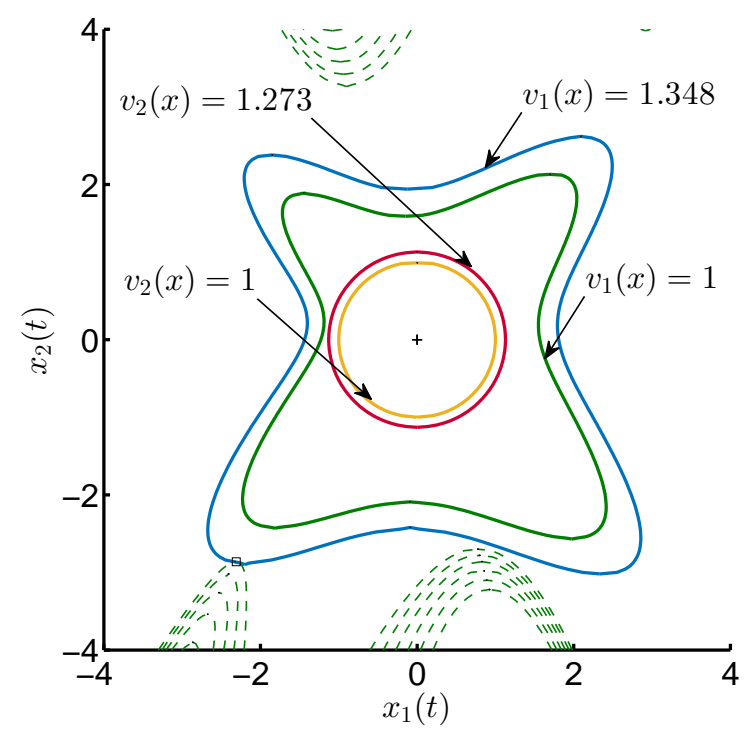

Fig. 2. The computational results: The solid blue line and red line indicate the bounds of the largest estimate of the RDA by using $v_{1}$ and $v_{2}$, respectively; the solid yellow line and green line indicate the bounds of the unitary sublevel set by using $v_{1}$ and $v_{2}$, respectively; the dashed lines indicate $\dot{v}(x, \tilde{\theta})=0$ with $k=5$ for some $\tilde{\theta} \in \tilde{\Theta}$.

can be calculated via a quasi-convex optimization consisting of a generalized eigenvalue problem for a selected rational Lyapunov function. Lastly, the tightness of the obtained lower bound can be established by a necessary and sufficient condition, which consists of checking the value of the derivative of Lyapunov function and checking whether a power vector is in a linear null space.

Future effort will be devoted to designing a less conservative convex approach to further enlarge the lower bound of $\mu^{*}$ by using variable rational Lyapunov functions, e.g., searching a Lyapunov function with the largest volume of the sublevel set $\mathcal{V}(c)$ (see [4], [23] for the case of polynomial Lyapunov functions). Moreover, we are interested to develop this approach combining with other robust verification methods, such as reachability analysis methods and contraction theory methods [29], [30].

\section{REFERENCES}

[1] H. K. Khalil and J. W. Grizzle. Nonlinear systems. Prentice Hall Upper Saddle River, 2002.

[2] A. Vannelli and M. Vidyasagar. Maximal Lyapunov functions and domains of attraction for autonomous nonlinear systems. Automatica, 21(1):69-80, 1985.

[3] C. W. Scherer and C. W. J. Hol. Matrix sum-of-squares relaxations for robust semi-definite programs. Mathematical Programming, 107(12):189-211, 2006.

[4] Z. Jarvis-Wloszek, R. Feeley, W. Tan, K. Sun, and A. Packard. Some controls applications of sum of squares programming. In Proceedings of Conference on Decision and Control, pages 4676-4681, 2003.

[5] W. Tan and A. Packard. Stability region analysis using polynomial and composite polynomial Lyapunov functions and sum-of-squares programming. IEEE Transactions on Automatic Control, 53(2):565570, 2008.

[6] S. Boyd, L. El Ghaoui, E. Feron, and V. Balakrishnan. Linear matrix inequalities in system and control theory. Society for industrial and applied mathematics, 1994.
[7] U. Topcu, A. Packard, and P. Seiler. Local stability analysis using simulations and sum-of-squares programming. Automatica, 44(10):2669 $-2675,2008$.

[8] B. Tibken. Estimation of the domain of attraction for polynomial systems via LMIs. In Proceedings of Conference on Decision and Control, pages 3860-3864, 2000.

[9] D. Henrion, J.-B. Lasserre, and J. Löfberg. Gloptipoly 3: moments, optimization and semidefinite programming. Optimization Methods \& Software, 24(4-5):761-779, 2009.

[10] G. Valmorbida and J. Anderson. Region of attraction analysis via invariant sets. In Proceedings of American Control Conference, pages 3591-3596, 2014.

[11] U. Topcu, A. K. Packard, P. Seiler, and G. J. Balas. Robust regionof-attraction estimation. IEEE Transactions on Automatic Control, 55(1):137-142, 2010.

[12] D. F. Coutinho, C. E. de Souza, and A. Trofino. Stability analysis of implicit polynomial systems. IEEE Transactions on Automatic Control, 54(5):1012-1018, 2009.

[13] G. Chesi. Estimating the domain of attraction for uncertain polynomial systems. Automatica, 40(11):1981-1986, 2004.

[14] G. Chesi. Robust domain of attraction: computing and controlling estimates with non-polynomial Lyapunov functions. In Proceedings of International Conference on Control Applications, pages 1086-1091, 2012.

[15] G. Chesi. Domain of attraction: analysis and control via SOS programming. Springer Science \& Business Media, 2011.

[16] S. Mastellone, P. F. Hokayem, C. T. Abdallah, and P. Dorato. Nonlinear stability analysis for non-polynomial systems. In Proceedings of the American Control Conference, pages 1725-1730, 2004.

[17] A. Papachristodoulou and S. Prajna. Analysis of non-polynomial systems using the sum of squares decomposition. In Positive polynomials in control, volume 312 of Lecture Notes in Control and Information Science, pages 23-43. Springer, 2005.

[18] E. Klipp, R. Herwig, A. Kowald, C. Wierling, and H. Lehrach. Systems biology in practice: concepts, implementation and application. John Wiley \& Sons, 2008.

[19] J. A. Jacquez. Compartmental analysis in biology and medicine. University of Michigan Press, 1985.

[20] G. Hexner. Stability of non-polynomial systems using differential inclusions and polynomial Lyapunov functions. In Proceedings of Conference on Decision and Control, pages 2946-2951, 2012.

[21] A. Saleme and B. Tibken. A new method to estimate a guaranteed subset of the domain of attraction for non-polynomial systems. In Proceedings of American Control Conference, pages 2577-2582, 2012.

[22] G. Chesi. Rational Lyapunov functions for estimating and controlling the robust domain of attraction. Automatica, 49(4):1051-1057, 2013.

[23] D. Han and M. Althoff. Control synthesis for non-polynomial systems: a domain of attraction perspective. In Proceedings of Conference on Decision and Control, pages 1160-1167, 2015.

[24] J. Bochnak, M. Coste, and M.-F. Roy. Real algebraic geometry. Springer, 1998.

[25] M. Putinar. Positive polynomials on compact semi-algebraic sets Indiana University Mathematics Journal, 42(3):969-984, 1993.

[26] A. Trofino and T. J. M. Dezuo. LMI stability conditions for uncertain rational nonlinear systems. International Journal of Robust and Nonlinear Control, 24(18):3124-3169, 2014.

[27] J. W. Brewer. Kronecker products and matrix calculus in system theory. IEEE Transactions on Circuits and Systems, 25(9):772-781, 1978.

[28] G. Chesi, A. Garulli, A. Tesi, and A. Vicino. LMI-based computation of optimal quadratic Lyapunov functions for odd polynomial systems. International Journal of Robust and Nonlinear Control, 15(1):35-49, 2005.

[29] M. Althoff and B. H. Krogh. Reachability analysis of nonlinear differential-algebraic systems. IEEE Transactions on Automatic Control, 59(2):371-383, 2014.

[30] D. Han and G. Chesi. Robust synchronization via homogeneous parameter-dependent polynomial contraction matrix. IEEE Transactions on Circuits and Systems I: Regular Papers, 61(10):2931-2940, 2014. 